\title{
Does the Reeh-Schlieder theorem violate relativistic causality?
}

\author{
Giovanni Valente \\ 1001 CL Cathedral of Learning, Pittsburgh, PA, 15260, USA
}

Received 17 October 2013

Received in revised form 29 April 2014

Accepted 24 May 2014

Available online 13 June 2014

\section{Introduction}

Relativistic causality is the requirement that causal processes cannot propagate faster than the speed of light. That is a constraint determining the causal structure of Minkowski spacetime in Einstein's theory of special relativity, which appears to be at variance with the quantum non-locality characterizing entangled states across spacelike separated systems. There lies a seeming inconsistency between quantum mechanics and special relativity. However, relativistic versions of quantum mechanics, such as Local Quantum Field Theory, have been successfully formulated. Even within such a framework, though, there remains some outstanding philosophical problems. A theorem by Reeh and Schlieder (1961) is a purely relativistic result in local quantum field theory, which is often regarded as raising a conflict with relativistic causality. Allegedly, under an operational interpretation, the theorem would entail non-local effects, in that by performing local operations within a certain region of spacetime one could instantaneously change the state of the field over another spacelike separated region. In particular, this may occur even in the vacuum. Puzzled reactions have been expressed by several physicists and philosophers of physics, most notably by Segal and Goodman (1965), who first referred to such non-local effects as "bizarre" and "physically quite surprising", as well as by Haag (1992), Redhead (1995), Fleming (2000), Fleming and Butterfield (1999), and Clifton and Halvorson (2001), among others. But does the Reeh-Schlieder theorem really violate relativistic causality? In this paper we claim that it does not. In fact, we wish to show that the alleged conflict is only apparent.

To be sure, various attempts to solve the conundrum have been developed in the literature. For instance, Redhead (1995) and Clifton and Halvorson (2001) suggested that not all local operations within a spacetime region retain full physical content, and hence the change of state of the field over another spacelike separated region ought to be traced back to a mere artifact of the mathematical formalism. More radically, Fleming (2000) and Fleming and Butterfield (1999) proposed to adopt a heterodox quantization scheme for quantum fields, so that to avoid the theorem and its consequences for the vacuum altogether. Instead, our purported solution rests on a careful analysis of the status of relativistic causality in local quantum field theory and its relation with the non-locality predicted by the operational interpretation of the Reeh-Schlieder theorem. Let us outline the structure of our argument, which we develop in the rest of the paper.

In special relativity, relativistic causality is meant to guarantee that an effect cannot temporally precede its cause. So, if it is violated, an event may actually occur before the event which causes 
it: one could then interfere with one's own past, thereby giving rise to causal paradoxes, such as the Grandfather Paradox. Yet, there are different ways to intend the prohibition of faster-than-light propagation of causal processes, depending on what one takes to be the relevant processes. In particular, such a constraint can be understood as a condition of no superluminal signalling or, alternatively, as a condition of no superluminal propagation of matter and energy carried by a field. In a recent paper Butterfield (2007) identified three main formulations of relativistic causality, corresponding to as many independent axioms of Local Quantum Field Theory: that is, Microcausality, the Spectrum Condition, and Local Primitive Caus-ality. Each axiom can be regarded as expressing the prohibition of superluminal propagation of causal processes intended either in the sense of signalling or in the sense of matter and energy carried by a field. Our argument shows that none of these formulations of relativistic causality is really in conflict with the Reeh-Schlieder theorem. For one, Microcausality is interpreted as assuring that signals cannot travel faster than light, at least when they are enacted by non-selective local operations. If so, given that such an axiom is invoked to make the case for the non-local effects implied by the theorem, the latter cannot entail a violation of no super-luminal signalling. On this point, though, more needs to be said about selective local operations. We argue that, even if one grants that such operations would enact superluminal signals, in the context of the Reeh-Schlieder theorem the resulting signals cannot be controlled, and hence one may not exploit them to interfere with one's own past, thereby evading the causal paradoxes arising from the failure of relativistic causality. As for the Spectrum Condition, it is regarded by Butterfield as "the most direct expression of the prohibition of spacelike processes" (p. 303), in that it requires that the spectrum of the energy-momentum operators of a field is confined to the future light cone. We observe that, since this condition is involved in the derivation of the ReehSchlieder theorem itself, the latter cannot entail any superluminal propagation of matter and energy in the relevant sense. Finally, Local Primitive Causality reflects the hyperbolic character of the underlying equations of motion. As we suggest here, it is the most appropriate formulation of no superluminal propagation of the field. Provably, it is fully consistent with the assumptions from which the Reeh-Schlieder theorem is proven. Thus, in the last analysis, we conclude that the Reeh-Schlieder theorem is not in conflict with relativistic causality at all.

The paper is organized as follows. We begin in Section 2 by reviewing the structure of Local Quantum Field Theory in its axio-matic formulation (Section 2). In particular, we emphasize in what sense the theory naturally embodies a concept of locality, which we contrast with the Bell-type non-locality due to the presence of entangled states. We then present the Reeh-Schlieder theorem and explain how it supposedly entails non-local effects (Section 3). In order to set its operational interpretation in precise terms, we cast the content of the theorem in terms of the notion of local operations: in doing so, we draw from a proposal by Clifton and Halvorson (2001), but we correct their formalization of the proper operations to associate with local observables. In Section 4 we take up the issue whether the theorem is at variance with the requirement that one cannot signal faster than light: in particular, we survey the attempt to explain away the nonlocal effects caused by selective operations by arguing that they are not fully physically significant; then, we argue that, even if one does not exclude superluminal signalling, the latter cannot be controlled in the context of the Reeh-Schlieder theorem, and hence it may not be used to give rise to causal paradoxes. In the last section, we conclude by discussing the condition of no super-luminal propagation of matter and energy carried by a field, and we propose that the most appropriate formulation of relativistic causality is captured by the axiom of Local Primitive Causality, which is fully consistent with the Reeh-Schlieder theorem.

\section{Causality and non-locality in Local Quantum Field Theory}

Local Quantum Field Theory has its standard axiomatic formulation in Rudolph Haag's (1992) seminal book, wherein the content of the theory is cast in terms of algebras of observables. For that reason, it is referred to as Algebraic Quantum Field Theory (AQFT). The structure of the relevant algebras is determined by a set of physically and mathematically motivated axioms. Here, we review the main axioms by placing particular emphasis on their relevance for the concepts of locality and causality.

Let us first recall some basic algebraic notions. The algebra $\mathcal{B}(\mathcal{H})$ represents the set of all bounded operators acting on the Hilbert space $\mathcal{H}$, which describes non-relativistic quantum systems. Then, any subalgebra $\mathcal{A} \subseteq \mathcal{B}(\mathcal{H})$ is a (concrete) $C^{*}$-algebra just in case it is closed under the operator norm topology, which requires that, if the sequence of operators $\left\{A_{n}\right\}$ in $\mathcal{A}$ converges in norm to some operator $A \in \mathcal{B}(\mathcal{H})$, i.e. $\left|A_{n}-A\right| \longrightarrow 0$, then $A$ belongs to $\mathcal{A}$. Von Neumann algebras arise as a special class of $C^{*}$-algebras. Indeed, the algebra $\mathcal{M}$ is a von Neumann algebra just in case it is closed under the strong operator topology (that is weaker than the operator norm topology), according to which, given the sequence of operators $\left\{A_{n}\right\}$ in $\mathcal{M}$, if for all vectors $\psi$ in $\mathcal{H}$ there exists some operator $A \in \mathcal{B}(\mathcal{H})$ such that $A n \psi \longrightarrow A \psi$, then $A$ belongs to $\mathcal{M}$. An algebra is called unital if it contains the identity transformation I on $\mathcal{H}$. Throughout the paper we always assume that the algebras we are dealing with are unital. As it turns out, the algebras describing relativistic quantum field systems in AQFT can be shown to be factors of von Neumann algebras of type III. For the sake of this paper, we do not really need to specify what factors of type III are, except for noting that they are proper subalgebras of

$\mathcal{B}(\mathcal{H})$. Let us also stress that we assume that the states defined on the relevant algebras are normal, that is countably additive.

The fundamental local character of AQFT is emphasized by Horuzhy (1990) in the following quote, which highlights the central role of causality constraints in the construction of the theory.

[T]here is a fundamental property which appears already at the early stage and deeply affects the conceptual (and, consequently, the mathematical) structure of algebraic quantum field theory. This property is locality, which is, in its turn, a combination of two properties: localization and causality. The former means that since any physical experiment takes place in a finite spacetime region, each physical quantity determined directly from the experiment is also associated with some region (localized in it). As to the latter property, one should keep in mind that no signal velocity can exceed the velocity of light, and no processes taking place in spacetime regions separated by spacelike intervals can affect each other (Einstein's causality principle). Consequently, each observable must also be causal, i.e. compatible with any other observable if their localization regions are mutually space-like. [Horuzhy (1990), p. 3]

According to Horuzhy, locality results from the combination of two further conditions, namely localization and causality. That offers a useful starting point for our discussion. So, let us see in detail how the intended conditions are built into the postulates of the theory.

The primitive object in the algebraic description of a quantum field system is the mapping

$\mathcal{O} \longrightarrow \mathcal{A}(\mathcal{O})$

from any bounded region $\mathcal{O}$ of Minkowski spacetime $M$, within which the system is thought of as being localized, onto a corresponding local algebra $\mathcal{A}(\mathcal{O})$. Localization guarantees that the latter contains all the observables one can measure in the corresponding spacetime region. Accordingly, all the elements of 
$\mathcal{A}(\mathcal{O})$ are referred to as local observables. The underlying intuition is that only the spatiotemporal localization of observables is necessary for a description of quantum fields. Yet, it is important to stress that the relevant physical information is encoded in the way in which the algebras of observables in the net $\{\mathcal{A}(\mathcal{O}) \mid \mathcal{O} \subset M\}$ are linked together. In fact, one does not need to specify what observables have physical significance: it is sufficient to investigate the net structure of the local algebras.

With the notion of localization at hand, one can now state the first axiom, expressing the fact that any observable that can be measured in a region $\mathcal{O}_{1}$ is also measurable in a larger region $\mathcal{O}_{2}$ containing $\mathcal{O}_{1}$. That is:

\section{Isotony: If $\mathcal{O}_{1} \subset \mathcal{O}_{2}$, then $\mathcal{A}\left(\mathcal{O}_{1}\right) \subset \mathcal{A}\left(\mathcal{O}_{2}\right)$}

If this holds for all regions of the spacetime manifold $M$, then there is a $\mathrm{C}^{*}$-algebra $\mathcal{A}$ arising as the inductive limit of the net of local algebras. The elements of such a quasi-local algebra can thus be uniformly approximated by local observables.

The second axiom captures the notion of causality referred to by Horuzhy. Let the causal complement of $\mathcal{O}$ be the region $\mathcal{O}^{\prime}$ comprising all the points in Minkowski spacetime that is spacelike separated from every point in $\mathcal{O}$. For any pair of regions $\mathcal{O}_{1}$ and $\mathcal{O}_{2}$, one requires the following:

\section{Microcausality: If $\mathcal{O}_{1} \subseteq \mathcal{O}_{2}{ }^{\prime}$, then $\left[\mathcal{A}\left(\mathcal{O}_{1}\right), \mathcal{A}\left(\mathcal{O}_{2}\right)\right]=\{0\}$.}

It means that, if $\mathcal{O}_{1}$ and $\mathcal{O}_{2}$ are spacelike separated regions, the corresponding local algebras are mutually commuting, that is $\mathcal{A}\left(\mathcal{O}_{1}\right) \subseteq \mathcal{A}\left(\mathcal{O}_{2}\right)^{\prime}$ (where the apex ' denotes the commutant of an algebra, namely the set of bounded of operators that commute with all elements of the algebra). That captures the idea that measurements of observables localized in spacelike separated regions are co-possible, in the sense that they do not disturb each other. Such a postulate is often called Einstein's principle of causality, as in Horuzhy's quote. On the basis of this interpretation, one can characterize microcausality as a condition of mutual independence between distant quantum field systems. Yet, Horuzhy suggests that it also bears direct causal significance as a prohibition of faster-than-light signals. As we argue in Section 4, though, the latter claim is true only under a rather special understanding of no superluminal signaling. For now, we just wish to point out that the condition of mutual commutativity between the algebras describing distant systems can actually be formulated in ordinary non-relativistic quantum mechanics too, although the localization of the observables would not be explicit there in that one lacks an association of the relevant algebras with spacetime regions. The next postulates of AQFT, instead, introduce purely relativistic constraints with no non-relativistic analogs.

Let $\mathcal{P}_{+}^{\dagger}$ be the identity-connected component of the Poincaré group. The third axiom assures the Poincare symmetry of the theory:

3. Relativistic Covariance: On the underlying Hilbert space $\mathcal{H}$ there exists a strongly continuous unitary representation $U(\Lambda, \alpha)$ of $\mathcal{P}_{+}^{\dagger}$, where $\Lambda$ a proper Lorentz transformation and $\alpha$ a four-vector in $M$, such that

$U(\Lambda, \alpha) \mathcal{A}(\mathcal{O}) U(\Lambda, \alpha)^{-1}=\mathcal{A}(\Lambda(\mathcal{O})+\alpha)$

for all regions $\mathcal{O}$ of Minkowski spacetime $M$.

Such a postulate embodies Einstein's principle of relativity, reflecting the Lorentz invariance of all physical laws: thus, the results of an experiment do not depend on the choice of any inertial reference frame. In particular, relativistic covariance includes the requirement of translation covariance.

There exist several different physically relevant representations. We here choose to work with the irreducible vacuum representation. Accordingly, all the local algebras in the net $\{\mathcal{A}(\mathcal{O}) \mid \mathcal{O} \subset M\}$ are assumed to act on the same (separable) Hilbert space $\mathcal{H}$, in which there is defined a distinguished unit vector $\psi_{0}$ corresponding to the vacuum that is invariant under $U(\Lambda, \alpha)$. One then places the following axiom:

4. Spectrum Condition: The spectrum of the self-adjoint generators of the strongly continuous representation of the translation sub-group of $\mathcal{P}_{+}^{\dagger}$ must lie in the closed forward light cone.

The spectrum of such generators is given by the physical interpretation of the global energy-momentum spectrum of the theory. Hence, this postulate corresponds to the requirement that energy is positive in every Lorentz frame. Assuming the spectrum condition is actually crucial in the derivation of the Reeh-Schlieder theorem. On the other hand, some authors have even identified it as an expression of relativistic causality in AQFT.

A further axiom is commonly made when dealing with the vacuum representation, that is

5. Weak Additivity: For all regions $\mathcal{O}$ in $M, \mathcal{A}$ is the smallest $\mathrm{C}^{*}$-algebra containing $\bigvee_{\alpha} \mathcal{A}(\mathcal{O}+\alpha)$

Since the relevant bounded regions can be chosen to be arbitrarily small, this postulate guarantees, in particular, that the spacetime continuum is completely homogeneous.

Typical bounded regions in AQFT are the double cones. Let us consider two points $x$ and $y$ of Minkowski spacetime such that the former lies in the forward light cone of the latter: an (open) double cone is the intersection of the causal future of $y$ and the causal past of $x$. Two spacelike separated double cones are said to be tangent if their closure intersects at a single point; else, they are strictly separated. For simplicity, we restrict our attention to the simplest example of double cones, namely the diamond regions, for which the said points $x$ and $y$ have the same time coordinate. Diamonds have the remarkable property that the commutant of their local algebra coincides with the local algebra associated with their causal complement, that is $\mathcal{A}(\mathcal{O})^{\prime}=\mathcal{A}\left(\mathcal{O}^{\prime}\right)$. In particular, local algebras associated with such regions naturally satisfy the axiom of Local Primitive Causality, which we regard as the appropriate expression of relativistic causality embodying the condition of no superluminal propagation of a field. We show this in the last section, where we also state the postulate explicitly.

To be sure, even when all the above axioms are satisfied, including those expressing causality and locality requirements, Bell's inequality is violated in Local Quantum Field Theory. In fact, in this setting the failure of Bell-type locality is even more dramatic than in non-relativistic quantum mechanics. For, let the normal global state $\phi$ be defined on the joint algebra $\mathcal{A}\left(\mathcal{O}_{1}\right) \vee$ $\mathcal{A}\left(\mathcal{O}_{2}\right)$ formed by the local algebras associated with the spacelike separated regions $\mathcal{O}_{1}$ and $\mathcal{O}_{2}$. Provably, an algebraic version of Bell's inequality fails for all global states across pairs of tangent regions as well as for all global states across pairs of strictly spacelike separated regions whose minimal Minkowski distance is not large enough (see Summers \& Werner, 1987, 1988). Moreover, Clifton and Halvorson (2000) demonstrated that, even in those cases where there exist some unentangled states, Bell's inequality is (maximally) violated by a norm dense set of global states, including the vacuum state, which implies that such global states

are all entangled across $\mathcal{A}\left(\mathcal{O}_{1}\right)$ and $\mathcal{A}\left(\mathcal{O}_{2}\right)$. As a matter of fact, this just arises as a consequence of the Reeh-Schlieder theorem. One may perhaps interpret the failure of Bell-type locality entailed by the theorem as a good indicator of a violation of relativistic causality. But is that really the case? 


\section{The operational interpretation of the Reeh-Schlieder theorem}

Before stating the Reeh-Schlieder theorem and explaining its content, we ought to recall some further relevant algebraic notions. A vector $\psi$ in the underlying Hilbert space $\mathcal{H}$ is said to be cyclic for $\mathcal{M}$ just in case the set $\{A \psi: A \in \mathcal{M}\}$ is norm dense in $\mathcal{H}$. That implies that, given every $\xi \in \mathcal{H}$, for any $\epsilon>0$ there exists an operator $A$ in $\mathcal{M}$ such that $\|A \psi-\xi\|<\epsilon$ : in other words, by applying a given element of the algebra to $\psi$ one can approximate as closely as one wishes any other vector. As a shorthand for this fact, in the rest of the paper we use the notation $\|A \psi-\xi\| \rightarrow 0$, although strictly speaking it is meaningless in the sense that there is no series that tends to zero here. Moreover, $\psi$ is said to be a separating vector for $\mathcal{M}$ just in case $A \psi=0$ implies $A=0$ for all $A \in \mathcal{M}$. As a consequence, for any two elements $A$ and $B$ of $\mathcal{M}$, if $(A-B) \psi=0$ then $A$ and $B$ must coincide. That means that $\psi$ is rich enough to distinguish different observables in the algebra. If there is a separating vector for an algebra, any normal state on such an algebra is a vector state (Kadison and Ringrose, 1997, Theorem 7.2.1): that is, for any normal state $\phi$ on $\mathcal{M}$, there is a vector $\xi \in \mathcal{H}$ such that $\phi=\phi_{\xi}$ where $\phi_{\xi}(\cdot)=\langle\xi,(\cdot) \xi\rangle /\langle\xi, \xi\rangle$. One can thus generate all states by means of the vectors belonging to the underlying Hilbert space. In the context of von Neumann algebras, a vector is separating for an algebra if and only if it is cyclic for its commutant.

The Reeh-Schlieder theorem can be derived from the above axioms of AQFT. In particular, it follows just from the requirement of translation invariance included in relativistic covariance, the spectrum condition and weak additivity. In its general formulation, the theorem is about vectors that are analytic in the energy, yet here we just present its statement for vectors of bounded energy. Specifically, a vector $\psi \in \mathcal{H}$ has bounded energy if $E([0, r]) \psi=$ $\psi$ for some $r<\infty$, with $E$ being the spectral measure of the global Hamiltonian of the field.

Reeh-Schlieder theorem: For all bounded regions $\mathcal{O}$ in $M$, if the vector $\psi$ has bounded energy, then $\psi$ is cyclic for the local algebra $\mathcal{A}(\mathcal{O})$.

Let us stress how strong and far-reaching such a result proves to be: it establishes that vectors of bounded energy are cyclic for any local algebra. In particular, since the global Hamitonian of the field takes on the value 0 in the vacuum vector $\psi_{0}$, the theorem entails the cyclicity of $\psi_{0}$ for all local algebras.

To illustrate the startling implications of the Reeh-Schlieder theorem for the issue of non-locality, let us begin by quoting a comment by Haag (1992), where the crucial role of the spectrum condition in its derivation is also emphasized:

the theorem tells us that for any chosen state vector $\xi$ one can always find an operator $A \in \mathcal{A}(\mathcal{O})$ which, applied to the vacuum, produces a state vector arbitrarily close to $\xi$. To achieve this the operator must judiciously exploit the small but non-vanishing long distance correlations which exist in the vacuum as a consequence of the spectral restrictions for energy-momentum in the theory. (p. 102, where the formalism has been suitably modified)

It would thus seem that, owing to the presence of entangled correlations in the vacuum, ${ }^{1}$ by operating within a certain spacetime region an experimenter could steer the vacuum into any other desired global state of the field. In order to achieve this, one needs to invoke microcausality. Indeed, while such an axiom is not

\footnotetext{
${ }^{1}$ Such correlations are actually known to decrease exponentially with the minimum Minkowski distance between spacelike separated regions, but they will never vanish completely (see Fredenhagen, 1985).
}

required in the proof of the theorem, it is indispensable to make the argument for the ensuing non-local effects. Since the vacuum vector $\psi_{0}$ is cyclic for the local algebra $\mathcal{A}\left(\mathcal{O}_{1}\right)$ associated with the diamond region $\mathcal{O}_{1}$, it is separating for the local algebra $\mathcal{A}\left(\mathcal{O}_{1}\right)^{\prime}$ associated with its causal complement $\mathcal{O}_{1}{ }^{\prime}$, and hence it is separating for the local algebra $\mathcal{A}\left(\mathcal{O}_{2}\right) \subseteq \mathcal{A}\left(\mathcal{O}_{1}\right)^{\prime}$ associated with any spacelike separated region $\mathcal{O}_{2}$. Likewise, since $\psi_{0}$ is cyclic for $\mathcal{A}\left(\mathrm{O}_{2}\right)$, it is separating for $\mathcal{A}\left(\mathcal{O}_{1}\right)$ as well. It then follows that $\psi_{0}$ is separating for the joint algebra $\mathcal{A}\left(\mathcal{O}_{1}\right) \vee \mathcal{A}\left(\mathcal{O}_{2}\right)$, which means that any global state of the field is generated by some vector $\xi$ in the underlying Hilbert space $\mathcal{H}$. Now, by exploiting the cyclicity of the vacuum for $\mathcal{A}\left(\mathcal{O}_{1}\right)$, for each $\xi$ one can find an observable $A$ localized in the region $\mathcal{O}_{1}$ such that $\left\|A \psi_{0}-\xi\right\| \rightarrow 0$. This implies that the global state $\phi_{\xi}$ on $\mathcal{A}\left(\mathcal{O}_{1}\right) \vee \mathcal{A}\left(\mathcal{O}_{2}\right)$ is approximated in norm $^{2}$ by the state $\phi_{A \psi_{0}}$ generated by the vector produced by hitting $\psi_{0}$ with the operator $A$, that is $\left\|\phi_{A \psi_{0}}-\phi_{\xi}\right\| \rightarrow 0$.

In particular, by operating in region $\mathcal{O}_{1}$ an experimenter could steer the vacuum into some global state of the field that looks like very different from the vacuum within the spacelike separated region $\mathcal{O}_{2}$. In fact, she could generate a state $\phi$ on $\mathcal{A}\left(\mathcal{O}_{1}\right) \vee \mathcal{A}\left(\mathcal{O}_{2}\right)$ whose restriction to the local algebra associated with $\mathcal{O}_{2}$ no longer coincides with the vacuum, in the sense that $\left.\phi\right|_{\mathcal{A}\left(\mathcal{O}_{2}\right)} \neq\left.\phi_{\psi_{0}}\right|_{\mathcal{A}\left(\mathrm{O}_{2}\right)}$. Such a non-local change of state would take place instantaneously, thereby raising an apparent conflict with relativistic causality. That is the puzzle we wish to resolve. ${ }^{3}$

To this extent, we point out another remarkable property of the vacuum established by Redhead (1995) as a corollary of the ReehSchlieder theorem, namely the existence of vacuum fluctuations. In Redhead's terminology, $\operatorname{Pr}_{\psi}(P=1)$ is the probability that a measurement represented by a projection $P$ yields a positive outcome in the vector-state $\psi$ : by construction, it corresponds to the value of the state $\phi_{\psi}$ evaluated on $P$. Redhead then proved that such a probability cannot vanish for any non-zero projection in the local algebra $\mathcal{A}(\mathcal{O})$. Consider a projection $P_{2}$ localized in the region $\mathcal{O}_{2}$ spacelike separated from $\mathcal{O}_{1}$, and suppose, for purpose of reductio ad absurdum, that $\operatorname{Pr}_{\psi_{0}}\left(P_{2}=1\right)=0$ in the vacuum. It follows straightforwardly that $\left\langle\psi_{0} \mid P_{2} \psi_{0}\right\rangle=\left\langle P_{2} \psi_{0} \mid P_{2} \psi_{0}\right\rangle=0$, and hence $P_{2} \psi_{0}=0$. Since $\psi_{0}$, being cyclic for $\mathcal{A}\left(\mathcal{O}_{1}\right)$, is also separating for $\mathcal{A}\left(\mathcal{O}_{2}\right)$, then $P_{2}$ must be equal to zero. By contraposition, one can thus infer that for any non-zero projection $P_{2}$ the probability $\operatorname{Pr}_{\psi \psi_{0}}\left(P_{2}=1\right)$ that the corresponding measurement yields a positive outcome in the vacuum $\psi_{0}$ cannot be zero. It means that, quite surprisingly, any possible outcome of measurements performed within a spacetime region has a finite, non-vanishing probability to occur in the vacuum. As Redhead put it, "[i]n the long run anything that is possible will happen in the vacuum" (p. 128), which illustrates his remark that, locally, the vacuum is "seething with activity" (p. 123). Actually, in our view, this point tends to obscure the possibility of evaluating alleged non-local, a-causal effects. In fact, if something happens in region $\mathcal{O}_{2}$, one cannot be sure that it is an effect of some action in region $\mathcal{O}_{1}$ instead of being just a spontaneous fluctuation of the vacuum. On the other hand, it would also be a quite surprising coincidence that such a random fluctuation occurs exactly at the same time when an experimenter performs a measurement somewhere else.

\footnotetext{
${ }^{2}$ The norm of a state $\phi$ on the von Neumann algebra $\mathcal{M}$ is defined by $\|\phi\| \equiv\left\{|\phi(A)|: A=A^{*} \in \mathcal{M},\|A\| \leq 1\right\}$.

${ }^{3}$ Actually, Haag himself calls the possibility of steering the vacuum into any global state a "superficial paradox", in that he argues that, if one is allowed to operate in such a way to involve just a physically reasonable expenditure of energy one could approximate only a subset of the state space. Yet, this requires one to appeal to the nuclearity condition, which does not hold in general. More importantly, though, the fact remains that one could still change the state of the field in a spacelike separated region.
} 
Be that as it may, the seeming violation of relativistic causality described above rests on an operational interpretation of the Reeh-Schlieder theorem. In the formulation adopted in the quote by Haag, though, the idea of operating within a spacetime region is cast in terms of the operators in the corresponding local algebra hitting the vacuum vector. However, the recent literature in quantum theory generalizes the notion of measurements in the language of operations (see Doplicher, Haag, \& Roberts, 1971, 1974; Hellwig \& Kraus, 1969, 1970; Kraus, 1983), which are maps over an algebra of observables rather than elements of the algebra. So, in order to make precise the operational interpretation of the theorem one ought to lift the discussion from the level of local observables to the level of local operations. We show how this can be done here below.

\subsection{From local observables to local operations}

Operations are the mathematical representative of actual operations, such as measurements, that an experimenter can perform on quantum systems. An operation is defined as a

completely positive linear map $T: \mathcal{M} \longrightarrow \mathcal{M}$ such that $T(I) \leq I$. It is a non-selective operation if it preserves the identity, that is

$T(I)=I$; otherwise, it is a selective operation. Furthermore, it is said to be projective just in case $T^{2}=T$. Given the initial state $\phi$ on $\mathcal{M}$, the operation-conditioned state is denoted by $T \phi=\not \circ T$, where $T^{*}$ is the dual of $T$ acting on the state space of $\mathcal{M}$. As we

deal with normal states, we consider just normal (i.e. $\sigma$-weakly continuous) operations, in that their dual transforms normal states into normal states. Operations mapping from $\mathcal{B}(\mathcal{H})$ onto itself can be given an explicit decomposition in terms of the Kraus representation theorem (Kraus, 1983): for any (normal) operation $T$ :

$\mathcal{B}(\mathcal{H}) \longrightarrow \mathcal{B}(\mathcal{H})$ there is at least one countable collection of Kraus operators $\left\{K_{i}\right\} \subseteq \mathcal{B}(\mathcal{H})$ such that

$T(\cdot)=\sum_{i} K_{i}^{*}(\cdot) K_{i}$

where $O \leq \sum_{i} K_{i}^{*} K_{i} \leq I$. If the sum is infinite, convergence is intended in the sense of the weak-* topology, which guarantees that $T$ is normal. For example, the Lüders rule for quantum measurements can be expressed in terms of operations. Let $P_{i}^{A \text { s }}$ s be the spectral projections of some discrete observable $A=\sum_{i} \lambda_{i} P_{i}^{A}$ in the algebra $\mathcal{M}$ describing a quantum system: a measurement of $A$ is represented by the non-selective (projective) operation $T_{P r o j}^{A}(\cdot)=\sum_{i} P_{i}^{A}(\cdot) P_{i}^{A}$; instead, the fact that a measurement of $A$ yields a specific outcome corresponding to the eigenvalue $\lambda_{i}$ is represented by the selective (projective) operation $T_{P_{i}}^{A}(\cdot)=P_{i}^{A}(\cdot) P_{i}^{A}$.

Local operations are a particular kind of operations expressing the idea of acting on a system without causing non-local effects on other distant systems. Here below we state the standard notion of local operations in its general algebraic formulation. Recall that the von Neumann algebra generated by $\mathcal{M}$ and its commutant $\mathcal{M}^{\prime}$ coincides with the algebra of all bounded operators on the underlying Hilbert space $\mathcal{H}$, that is $\mathcal{M} \vee \mathcal{M}^{\prime}=\mathcal{B}(\mathcal{H})$. One can then define a local operation admitting a Kraus decomposition as follows:

Local operations: A local operation in $\mathcal{M}$ is an operation $T$ : $\mathcal{B}(\mathcal{H}) \longrightarrow \mathcal{B}(\mathcal{H})$ whose representation is given in terms of Kraus operators $K_{i} \in \mathcal{M}$.

Having this formulation at hand, we now proceed to spell out the connection of the thus-defined local operations with the content of cyclicity of a vector $\psi$ for the algebra $\mathcal{M}$. Subsequently, we explain in what sense such operations can be thought of as embodying a locality requirement by showing how it applies to the local algebras of AQFT.

Clifton and Halvorson (2001) stressed the importance of cast-ing the meaning of cyclicity into the language of operations in order to clarify the operational interpretation of the Reeh-Schlieder theorem. To this extent, one needs to construct a local operation that corresponds to the action of the operator $A$ on the cyclic vector $\psi$ for $\mathcal{M}$ such that $\left\|\phi_{A \psi}-\phi_{\xi}\right\| \rightarrow 0$ for any chosen state defined by vector $\xi$ in the underlying Hilbert space $\mathcal{H}$. Clifton and Halvorson submitted the following proposal for the sought-after operation:

$\phi_{A \psi}$ is just the state one gets by applying the pure operation given by the Kraus operator $A /\|A\| \in \mathcal{M}$ to $\phi_{\psi}$. If follows that if $\psi$ is cyclic for $\mathcal{M}$, one can get arbitrarily close in norm to any other pure state of $\mathcal{B}(\mathcal{H})$ by applying an appropriate pure local operation in $\mathcal{M}$ to $\phi_{\psi}$. [Clifton and Halvorson (2001), p. 17, where the formalism has been suitably modified]

While Clifton and Halvorson's intuition is certainly on the right track, we would like to observe that their suggested choice of local operation is not correct. For, let us consider an observable $A$ in the algebra $\mathcal{M}$ for which $\psi$ is cyclic. If the Kraus operator associated with the action of $A$ on $\psi$ were given by $A /\|A\|$, the corresponding operation would take the form $T_{A \psi}(\cdot)=A^{*} /\|A\|(\cdot) A /\|A\|$. That is indeed a pure local operation in $\mathcal{M}$. In order to assure that such an operation would produce the state generated by the vector $A \psi$ when being applied to $\phi_{\psi}$, one ought to argue that $T_{A \psi}^{*} \phi_{\psi}$ is close to $\phi_{A \psi}$ in norm. Nevertheless, an explicit calculation shows that one obtains

$T_{A \psi}^{*} \phi_{\psi}(\cdot)=\frac{\left\langle\psi, \frac{A^{*}}{\|A\|}(\cdot) \frac{A}{\|A\|} \psi\right\rangle}{\langle\psi, \psi\rangle}=\frac{\langle A \psi,(\cdot) A \psi\rangle}{\|A\|^{2}\langle\psi, \psi\rangle}$

and this is in general not equal to $\phi_{A \psi}(\cdot)=\langle A \psi,(\cdot) A \psi\rangle /\langle A \psi, A \psi\rangle$. For instance, let $A$ be a non-zero projector onto a subspace orthogonal to $\psi$ : then $\langle A \psi, A \psi\rangle=0$ while $\|A\|^{2}\langle\psi, \psi\rangle \neq 0$, and thus the denominators in the expressions for $\phi_{A \psi}$ and $T_{A \psi}^{*} \phi_{\psi}$ do not coincide. Instead, it turns out that the correct expression for the local operation in $\mathcal{M}$ corresponding to the action of $A$ on $\psi$ is given by

$T_{A \psi}(\cdot)=\frac{A^{*}(\cdot) A}{\phi_{\psi}\left(A^{*} A\right)}$

So, at least when $A$ is a positive operator such that $A^{*} A \leq I$, one can conclude that the appropriate choice for the Kraus operator is $A\|\psi\| /\|A \psi\|$. Obviously, this definition would coincide with the one provided by Clifton and Halvorson just in the trivial case in which $A$ is the identity $I$ in the algebra $\mathcal{M}$.

Next, we turn to the locality requirement characterizing the notion of local operations. In order to make it more conspicuous from a physical point of view, we discuss its meaning in the context of AQFT. Let the von Neumann algebra $\mathcal{M}$ be the local algebra $\mathcal{A}\left(\mathcal{O}_{1}\right)$ associated with a region $\mathcal{O}_{1}$. Its causal complement $\mathcal{O}_{1}^{\prime}$ is associated with the algebra $\mathcal{A}\left(\mathcal{O}_{1}^{\prime}\right)$, which by the diamond property corresponds to $\mathcal{A}\left(\mathcal{O}_{1}\right)^{\prime}$. Accordingly, a local operation $T$ in $\mathcal{A}\left(\mathcal{O}_{1}\right)$ is an operation defined on $\mathcal{A}\left(\mathcal{O}_{1}\right) \vee \mathcal{A}\left(\mathcal{O}_{1}\right)^{\prime}=\mathcal{B}(\mathcal{H})$. The fact that its representation is given in terms of Kraus operators $K_{i}$ taken from the algebra $\mathcal{A}\left(\mathcal{O}_{1}\right)$ guarantees that one is operating within the region $\mathcal{O}_{1}$. The idea that performing such an operation does not have non-local effects is captured by the requirement that $T$ does not change the state of the field over the causal complement of $\mathcal{O}_{1}$, in the sense that for any global state $\phi$ its restriction to $\mathcal{A}\left(\mathcal{O}_{1}\right)^{\prime}$ remains invariant, i.e. $\left.T^{*} \phi\right|_{\mathcal{A}\left(\mathcal{O}_{1}\right)^{\prime}}=\left.\phi\right|_{\mathcal{A}\left(\mathcal{O}_{1}\right)^{\prime}}$. Provably, for nonselective operations such a locality requirement is always satisfied. ${ }^{4}$ Thus, a fortiori, a non-selective local operation in $\mathcal{A}\left(\mathcal{O}_{1}\right)$ does

\footnotetext{
${ }^{4}$ For this reason, the thus-defined (non-selective) local operations are labeled absolutely local operations by Valente (2013). However, if one wishes to consider (non-selective) local operations in $\mathcal{A}\left(\mathcal{O}_{1}\right)$ that leave invariant the state of the field just over the region $\mathcal{O}_{2}$, rather than over the entire causal complement $\mathcal{O}_{1}^{\prime}$, one ought to construct different maps, called relatively local operations. Actually, since
} 
not change the state of the field over any region $\mathcal{O}_{2}$ spacelike separated from $\mathcal{O}_{1}$.

However, notice that the above definition of local operations includes selective operations as well. If $T$ is a selective local operation in $\mathcal{A}\left(\mathcal{O}_{1}\right)$, then $T(B)=T(I) B$ for all $B \in \mathcal{A}\left(\mathcal{O}_{1}\right)^{\prime}$. That implies that, for any global state $\phi, T^{*} \phi(B)=\phi(T(I) B) / \phi(T(I))$, which is certainly different from $\phi(B)$ for some observable $B$ localized in $\mathcal{O}_{1}{ }^{\prime}$. Therefore, selective local operations do change the state of the field over the causal complement of the region $\mathcal{O}_{1}$. It follows that $\left.T^{*} \phi\right|_{\mathcal{A}\left(\mathcal{O}_{2}\right)} \neq\left.\phi\right|_{\mathcal{A}\left(\mathcal{O}_{2}\right)}$ when the region $\mathcal{O}_{2}$ is spacelike separated from $\mathcal{O}_{1}$. Whether or not such a change of state counts as a violation of locality depends on one's physical interpretation of local operations. We shall return to this issue in Section 4.1.

Having formulated the operational interpretation of the ReehSchlieder theorem in precise terms, we can now tackle its alleged conflict with relativistic causality. We first address the question whether it violates the requirement of no superluminal signalling. Then, we discuss whether it infringes on the relativistic constraint of no superluminal propagation of matter and energy carried by a quantum field.

\section{No superluminal signalling}

According to the quotation from Horuzhy in Section 2, the rationale for ascribing causal significance to the axiom of microcausality is two-fold: first, it would guarantee the co-possibility of physical processes, such as measurements, taking place at the same time in different spacelike separated regions; second, it would impose constraint forbidding signals to travel faster than light. As we already emphasized, under the first characterization microcausality appears as a condition of mutual independence between distant quantum field systems. Yet, there is a hierarchy of independence conditions formulated in the literature depending on what one takes to be the relevant physical process (see Summers, 1990 for a thorough discussion), and microcausality is not even the strongest among such conditions. For instance, Rédei and Summers (2008) proposed a condition of operational independence demanding that general operations performed on spacelike separated regions do not disturb each other and can be performed simultaneously. Such a requirement is satisfied when the split property holds. The latter is a strengthening of microcausality, which also entails a condition of statistical independence for spacelike separated quantum fields. ${ }^{5}$ However that may be, it is only the second characterization of microcausality as a prohibition of faster-than-light signals that would enforce a direct connection with relativistic causality. But why should the requirement of mutual commutativity of the local algebras associated with spacelike separated regions assure no superluminal signalling?

This issue is highly sensitive to what one takes to constitute a signalling procedure, which in general requires one to specify some mechanisms for both the emission and the reception of a signal, as well as a means of transmission (Weinstein, 2006 offers a review of various options). Setting these concerns aside, the standard answer to the above question is based on a theorem by Schlieder (1968) involving the quantum-mechanical Lüders rule. To illustrate the operational content of Schlieder's result in the context of AQFT, let us consider the operation $T_{P r o j}^{A}(\cdot)=\sum_{i} P_{i}^{A}(\cdot) P_{i}^{A}$ representing the measurement of some discrete observable $A$ localized in region $\mathcal{O}_{1}$, whose spectral decomposition is given in terms of the projections $P_{i}^{A \text { s }}$ s. The theorem guarantees that such an operation does not change the state of the field on the spacelike separated region $\mathcal{O}_{2}$, i.e. $\left.T_{\text {Proj }}^{A *} \phi\right|_{\mathcal{A}\left(\mathcal{O}_{2}\right)}=\left.\phi\right|_{\mathcal{A}\left(\mathcal{O}_{2}\right)}$ for any initial global state $\phi$, if and only if the local algebras $\mathcal{A}\left(\mathcal{O}_{1}\right)$ and $\mathcal{A}\left(\mathcal{O}_{2}\right)$ are mutually commuting. Therefore, no possible signal enacted by performing a measurement in $\mathcal{O}_{1}$ could be detected in $\mathcal{O}_{2}$, in that the state of the field within the latter region remains invariant. One may actually object that here the relevant notion of signal is restricted to non-selective projective operations. Yet, the fact that, as we explained at the end of the previous section, all nonselective local operations in $\mathcal{A}\left(\mathcal{O}_{1}\right)$ leave unchanged the restriction of any global state $\phi$ to $\mathcal{A}\left(\mathrm{O}_{2}\right)$ provides an operational generalization of Schlieder's theorem. Accordingly, microcausality would yield necessary and sufficient conditions for no superluminal signalling, at least under the present notion of local operations. ${ }^{6}$

To come back to the Reeh-Schlieder theorem, one may think that these considerations lead one to dissolve the conflict with relativistic causality intended as a constraint of no faster-than-light signalling. Nevertheless, we believe that one is not out of the woods yet. For one, although microcausality is not needed to derive the theorem, its puzzling non-local effects would arise just in case one adds such an axiom. This point is hard to square with the claim that microcausality prohibits superluminal signals. Furthermore, such a claim can be maintained only if one considers non-selective operations. Indeed, we know that selective opera-tions performed in one region change the state of the field within its causal complement. To cope with this fact there are two main strategies available: either one tries to explain away the apparent non-local effects by arguing that those local operations that would cause an instantaneous change of state in a distant region do not retain full physical content; or one shows that, even if the possibility of superluminal signaling is not excluded, the resulting signals are necessarily uncontrollable, and thus they may not be used to give rise to causal paradoxes. In the rest of this section, we examine such options in that order.

\subsection{What local operations are physical?}

When discussing the operational interpretation of the ReehSchlieder theorem, Redhead (1995) proposed a distinction between physical and mathematical operations in order to solve the puzzle of non-locality arising from the cyclicity of the vacuum. His classification was developed at the level of local observables, rather than local operations. He listed the operations of "making measurements, selecting subensembles according to the outcomes of measurements, and mixing ensembles with probabilistic weights" ( $p$. 128) as physical; whereas he characterized the operation of producing superpositions of states as mathematical. Then, on the basis of an analogy with the singlet state in the non-relativistic case, he observed that one could only approximate all possible global states if one also applies such mathematical operations. While this analysis is correct, Redhead failed to recognize that a local operation corresponding to an observable localized in a given region can change the state of a field over another spacelike separated region just in case it is selective.

By stressing this point, Clifton and Halvorson (2001) put forward a different distinction. For them, only non-selective operations retain full physical significance, whereas selective operations contain also a conceptual component. In fact, a non-selective

${ }^{5}$ Summers (2009) offers an illuminating review of the split property as a sufficient condition for microscopic subsystems to be conceived as independent.

${ }^{6}$ With regard to this point, it should be stressed that such a claim holds true just for absolutely local operations (see footnote 4). In fact, the existence of relatively local operations in AQFT is assured under the split property, as it was proven by Rédei \& Valente (2010), and hence microcausality is not sufficient for a prohibition of faster-than-light signals enacted by such operations. 
operation is conceived as representing the actual, physical interaction between a quantum system and the measurement apparatus, whereby an ensemble of copies of the system is decomposed into different subensembles corresponding to all the possible outcomes of the measurement. Instead, a selective operation ought to be understood as a combination of a non-selective operation, due to the physical act of performing a measurement, and the additional purely conceptual operation of singling out the subensemble corresponding to a specific outcome of such a measurement, while discarding the other non-relevant subensembles. Accordingly, in our example of application of the Lüders rule, $T_{P r o j}^{A}$ enacts a partition of the ensemble into as many subensembles as the possible outcomes of a measurement of the observable $A$ in $\mathcal{A}\left(\mathcal{O}_{1}\right)$, whereas $T_{P_{i}}^{A}$ further selects the subensemble corresponding to the specific eigenvalue $\lambda_{i}$. Since non-selective operations performed in one region cannot change the state of the field over any other region in its causal complement, the change of state caused by a selective local operation is to be traced back to its conceptual component. By applying their proposed distinction to the operational interpretation of the Reeh-Schlieder theorem, Clifton and Halvorson concluded that:

the correct way to view the physical content of cyclicity is that changes in the global state are partly due to an experimenter's ability to perform a generalised measurement [in region $\mathcal{O}_{1}$ ], and partly due (pace Redhead) to the purely conceptual operation of selecting a subensemble based on the outcome of the experimenter's measurement together with the consequent "change" in the state [over region $\mathcal{O}_{2}$ ] via the EPR correlations. [p. 19, where the text has been suitably modified]

The upshot of their analysis is that the change of state of the field within region $\mathcal{O}_{2}$ being due to the conceptual component of a selective operation in region $\mathcal{O}_{1}$ ought not to be regarded as a real, physical change. Rather, it just reflects a mere change of knowledge of the experimenter in light of learning that her measurement has elicited a certain outcome. This offers an argument in support of the claim that selective operations whose representation is given in terms of Kraus operators localized in $\mathcal{O}_{1}$ should count as local operations in $\mathcal{A}\left(\mathcal{O}_{1}\right)$, notwithstanding their seeming violation of the locality requirement for local operations, which we described at the end of Section 3.1.

Admittedly, Clifton and Halvorson's purported interpretation of selective operations as being partially unphysical is rather controversial. In fact, as they themselves observed, it rests on an alleged solution of the infamous measurement problem according to which quantum states are ascribed an epistemic content. Nonetheless, they do not offer any further independent argument in favor of this position. To be sure, the measurement problem could be resolved on the basis of a complete description of the interaction between physical systems and measurement apparata, which is not currently available in AQFT. Yet, one ought to recognize that the present situation is in no way worse than that in ordinary quantum mechanics. On the positive side, though, we now wish to argue that in the relativistic case there is a more advantageous prospect to cope with the threat of superluminal signals that is made by selective operations, at least in the context of the Reeh-Schlieder theorem.

\subsection{Can signals be controlled?}

Bell (1975) argued that the quantum non-locality due to the violation of Bell's inequality would not infringe on relativity theory, in so far as superluminal signals are not controllable. This point is further developed by Maudlin (1994). Yet, various authors, such as Jones and Clifton (1993), Berkovitz (1998a, 1998b) and
Weinstein (2006), objected that the intended notion of controllability suffers from being vague. In fact, in ordinary quantum mechanics the issue whether or not faster-than-light signals, associated with the failure of outcome independence in the decomposition of Bell-type locality, can be controlled remains unsettled. On the contrary, there is a sense in which the kind of superluminal signalling predicted by the operational interpretation of the Reeh-Schlieder theorem is provably uncontrollable. We would like to offer two reasons in support of this thesis.

The first reason rests on topological considerations. Recall that, in the spirit of cyclicity, the sense in which one can steer the vacuum into any desired global state of the field by means of local operations is that of an approximation in norm. According to the uniform topology, two states $\phi$ and $\phi^{\prime}$ on the von Neumann algebra $\mathcal{M}$ approximate each other in norm, i.e. $\left\|\phi^{\prime}-\phi\right\| \rightarrow 0$, just in case they dictate close expectation values for all observables in $\mathcal{M}$. Yet, this does not mean that such states coincide: they are just topologically indistinguishable. Now, suppose that, for the sake of superluminal communication, an experimenter sets herself to change the vacuum state $\phi_{\psi_{0}}$ into a global state $\phi$ which looks like different from the vacuum within a region $\mathcal{O}_{2}$ spacelike separated from the region $\mathcal{O}_{1}$ where the experimenter is operating, so that $\left.\phi\right|_{A\left(\mathrm{O}_{2}\right)} \neq\left.\phi_{\psi}\right|_{\mathcal{A}\left(\mathrm{O}_{2}\right)}$. To accomplish this task, she can certainly choose an appropriate selective local operation $T_{A \psi 0}$ in $\mathcal{A}\left(\mathcal{O}_{1}\right)$ such that $\left\|T_{A \psi_{0}}^{*} \phi_{\psi_{0}}-\phi\right\| \rightarrow 0$, in agreement with the operational interpretation of the Reeh-Schlieder theorem. Nevertheless, once she performed her operation, although she has changed the state of the field over the region $\mathrm{O}_{2}$, she is not entitled to conclude that she has effectively obtained the sought-after global state $\phi$. In fact, from a purely topological point of view, she has no means to determine whether this is the case. Only if she could do so, may she have full control on her signalling procedure.

The second reason is a refinement of an argument made by Redhead (1995). As a corollary of the Reeh-Schlieder theorem, he proved that, for all projections $P_{2} \in \mathcal{A}\left(\mathrm{O}_{2}\right)$, there is a projection $P_{1}$ in $\mathcal{A}\left(\mathcal{O}_{2}\right)$ such that the probability that a local measurement of $P_{2}$ yields a positive outcome in the vacuum given that the measurement of $P_{1}$ has yielded a positive outcome is close to one, that is $\operatorname{Pr}_{\psi_{0}}\left(P_{1}=1 \mid P_{2}=1\right)=1-\epsilon$ for any arbitrarily small real number $\epsilon>0$. However, he also showed that, as a consequence of the axioms of AQFT, such a conditional probability can never be equal to 1 . If one casts this point in the language of operations, one can see how superluminal signals, if they are possible at all, cannot really be controlled. By construction, the conditional probability corresponds to the value of the operation-conditioned state $T_{P_{1} \psi_{0}}^{*} \phi_{\psi_{0}}$ for $P_{2}$. Now, let us assume, for purpose of reductio ad absurdum, that $T_{P_{1} \psi_{0}}^{*} \phi_{\psi_{0}}\left(P_{2}\right)$ is equal to 1 . That implies $T_{P_{1} \psi_{0}}^{*} \phi_{\psi_{0}}\left(I-P_{2}\right)=0$ where the projection $I-P_{2}$ belongs to $\mathcal{A}\left(\mathcal{O}_{2}\right)$ too. It follows straightforwardly that $\left\langle P_{1} \psi_{0} \mid\left(I-P_{2}\right) P_{1} \psi_{0}\right\rangle=\left\langle\psi_{0} \mid P_{1}^{*}\left(I-P_{2}\right) P_{1} \psi_{0}\right\rangle=0$, and by microcausality $\left\langle\psi_{0} \mid P_{1}^{*} P_{1}\left(I-P_{2}\right) \psi_{0}\right\rangle=0$. One can thus infer that $P_{1}\left(I-P_{2}\right) \psi_{0}$ must be equal to zero, and since $\psi_{0}$ is separating for $\mathcal{A}\left(\mathcal{O}_{1}\right) \vee \mathcal{A}\left(\mathcal{O}_{2}\right)$ one has $P_{1}\left(I-P_{2}\right)=0$. However, this is in flat contradiction with the Schlieder property in AQFT, which establishes that for any two non-null elements of the mutually commuting local algebras $\mathcal{A}\left(\mathcal{O}_{1}\right)$ and $\mathcal{A}\left(\mathcal{O}_{2}\right)$, respectively, their product cannot be zero. So, one concludes $T_{P_{1} \psi_{0}}^{*} \phi_{\psi \mu_{0}}\left(P_{2}\right) \neq 1$. It means that one cannot control the long distance correlations present in the vacuum in such a way as to trigger a positive outcome of a measurement of $P_{2}$ by performing a measurement of $P_{1}$. That ought to be contrasted with the non-relativistic case, where, as Redhead himself pointed out, if one employs the singlet state instead of the vacuum state, the above conditional probability can be made equal to one for a suitable choice of projections.

The upshot is that, if an experimenter has no control on the signals she purports to send, she cannot reliably interfere with her past, even if such signals could in principle travel faster than light. Hence, causal paradoxes may not arise. Indeed, as Bell intimated, 
uncontrollable superluminal signaling is not really at odds with the spirit of Einstein's theory of special relativity. For that matter, this claim is further corroborated by the recognition that in a field theory, such as special relativity, relativistic causality has a more natural understanding as a constraint on the speed of propagation of matter and energy carried by a field, rather than on the transmission of (non-material) messages. How the condition of no superluminal propagation of a field can be formulated in local quantum field theory is the subject of next section.

\section{No superluminal propagation of a field}

In order to complete our argument that the Reeh-Schlieder theorem does not violate relativistic causality, we now need to show that it does not entail any superluminal propagation of a quantum field. There are two main candidates to express the constraint that matter and energy cannot travel faster than light in local quantum field theory: the Spectrum Condition and Local Primitive Causality. Provably, none of the them is in conflict with the Reeh-Schlieder theorem.

Let us begin by evaluating the Spectrum Condition, which Butterfield (2007) suggests is a direct expression of relativistic causality in AQFT. The rationale for such a claim can be given along the following lines. Let us stress that, on the basis of the StoneNaimark-Ambrose-Godement theorem, the strongly continuous unitary implementation $T(\alpha):=U(1, \alpha)$ of the translation group of Minkowski spacetime $M$ can be written as

$T(\alpha)=e^{i\left(\alpha_{0} P_{0}-\alpha_{1} P_{1}-\alpha_{2} P_{2}-\alpha_{3} P_{3}\right)}$

in terms of the commuting self-adjoint operators $P_{a}$, with $a=0$, $1,2,3$. The Spectrum Condition requires that $P_{0} \geq 0$ and $P_{0}^{2}-P_{1}^{2}-$ $P_{2}^{2}-P_{3}^{2} \geq 0$, thereby ensuring the positivity of energy in all inertial frames. Then, if the generators $P_{a}$ of $T(\alpha)$ are identified with the energy-momentum operators of a field, the spectrum of such operators ought to lie in the forward light cone. It thus seems that the energy-momentum flow of the field is bound to propagate along non-spacelike trajectories. Yet, one may still raise doubts about whether this is necessarily the case. Indeed, Earman and Valente (2014) observed that, even if the spectrum condition holds, the positivity of energy is not guaranteed at small scales: for instance, Epstein et al. (1965) showed that the value of the energy density of quantum fields can be negative at some points of Minkowski spacetime. $^{7}$

However that may be, as it is emphasized in the quotation from Haag at the beginning of Section 3, the Spectrum Condition is a crucial ingredient in the derivation of the Reeh-Schlieder theorem. To make this point more explicit, such an assumption implies that, whenever a vector $\psi$ in the underlying Hilbert space $\mathcal{H}$ is analytic for the generator $P_{0}$ of time translations, and therefore it is a vector of bounded energy, the vector $T(\alpha) \psi$ has an analytic continuation, so that by means of the axiom of weak additivity one can then show that $\psi$ is cyclic for the local algebra $\mathcal{A}(\mathcal{O})$ of any arbitrary region $\mathcal{O}$. This means that, if one wishes to express the prohibition of faster-than-light propagation of matter and energy carried by a field by means of the spectrum condition, there cannot be any possible conflict between the Reeh-Schlieder theorem and relativistic causality, since the former would just be a consequence of the latter.

\footnotetext{
7 Incidentally, this would also prevent one from finding direct quantum analogs of the Dominant Energy Condition, formulated as a constraint that the flow of energy and matter cannot travel faster than light. See Earman (2014) for an argument that such a condition is not necessary for no superluminal propagation of a field.
}

In our view, though, it is the axiom of Local Primitive Causality that ought to be regarded as the most appropriate expression of relativistic causality in AQFT. In order to state it explicitly, let $\mathcal{D}(\mathcal{O})$ be the domain of dependence of the region $\mathcal{O}$, that is the union of $\mathcal{D}^{+}(\mathcal{O})$ and $\mathcal{D}^{-}(\mathcal{O})$, namely the future domain of dependence and the past domain of dependence of $\mathcal{O}$, respectively: specifically, $\mathcal{D}^{+}(\mathcal{O})$ is defined as the region comprising all points $p$ in $M$ such that every past inextendible causal curve passing through $p$ intersects $\mathcal{O}$, whereas $\mathcal{D}^{-}(\mathcal{O})$ is defined analogously. The axiom then reads as follows:

\section{Local Primitive Causality: $\mathcal{A}(\mathcal{O})=\mathcal{A}(\mathcal{D}(\mathcal{O})$ ) for any $\mathcal{O}$}

It means that all one can measure within a given spacetime region, namely the observables localized in it, fixes the local algebra associated with its causal domain of dependence. To put it informally, whatever happens in $\mathcal{D}^{+}(\mathcal{O})$ is determined just by what happens in $\mathcal{O}$ (likewise, whatever happens in $\mathcal{O}$ is determined by what happens in $\mathcal{D}^{-}(\mathcal{O})$ ). The above condition trivially holds for diamond regions, in that they coincide with their causal domain of dependence, that is $\mathcal{O}=\mathcal{D}(\mathcal{O})$. Local Primitive Causality is provably satisfied by many models of concrete quantum fields, such as the Klein-Gordon field and the Dirac field (Dimock, 1982). Indeed, it reflects the hyperbolic character of the underlying equations of motions. As such, this postulate captures the requirement of no superluminal propagation of a field, and hence it assures that matter and energy carried by the field cannot travel faster than light. All relevant physical processes must then evolve in the course of time within light cones.

To enforce this claim, we wish to stress that Local Primitive Causality entails a condition of Local Quantum Determinism that Earman and Valente (2014) formulated as a direct generalization of the constraint of no faster-than-light propagation for classical field theories to relativistic quantum field theory. The idea is that classical fields obey symmetric, quasi-linear, hyperbolic partial differential equations (pdes), whose initial value formulation has a nice locality property: namely, given a Cauchy surface $\Sigma$ of Minkowski spacetime, the initial data on any subset $\mathcal{S} \subset \Sigma$ uniquely determines a solution of the relevant pdes over the domain of dependence $\mathcal{D}(\mathcal{S})$ of $\mathcal{S}$. That guarantees no superluminal propagation of a classical field defined on the manifold $M$ (see

Geroch, 2011 for a detailed discussion). The quantum analog of such a locality property in the context of AQFT is given by

Local Quantum Determinism: For any $\mathcal{O} \subset M$ and any pair of states $\phi$ and $\phi^{\prime}$ on the quasi-local algebra $\mathcal{A}=\overline{\{\mathcal{A ( O )} \mid \mathcal{O} \subset M\}}$, if $\left.\phi\right|_{\mathcal{A}(\mathcal{O})}=\left.\phi^{\prime}\right|_{\mathcal{A}(\mathcal{O})}$ then $\left.\phi\right|_{\mathcal{A}(\mathcal{D}(\mathcal{O}))}=\left.\phi^{\prime}\right|_{\mathcal{A}(\mathcal{D}(\mathcal{O}))}$

In fact, this condition requires that, whenever two states coincide over the local algebra associated with a given region, in the sense that they assign the same value to any observable localized in it, then they cannot differ with respect to any observable belonging to the local algebra associated with its causal domain of dependence. In other words, any state of the field over the region $\mathcal{O}$ uniquely determines its extension over $\mathcal{D}(\mathcal{O})$. Accordingly, once the state of the field over a region $\mathcal{O}_{2}$ is fixed, nothing that an experimenter can do in the spacelike separated region $\mathcal{O}_{1}$ outside $\mathcal{D}\left(\mathrm{O}_{2}\right)$ would have any instantaneous detectable effect within $\mathcal{O}_{2}$ (or even within its entire causal domain of dependence). It follows straightforwardly from the above definition that Local Primitive Causality is sufficient for Local Quantum Determinism to hold, and hence for no superluminal propagation of a relativistic quantum field to be satisfied.

In order to ensure that the Reeh-Schlieder theorem is fully consistent with relativistic causality, we wish to note that Local Primitive Causality is completely independent from the other main axioms of AQFT, as it has been demonstrated by Haag and Schroer (1962). 
In particular, it can hold together with those postulates from which the theorem and its alleged non-local effects are derived, namely relativistic covariance, the spectrum condition, weak additivity and microcausality. Therefore, in the last analysis, we conclude that the Reeh-Schlieder theorem does not violate relativistic causality at all.

\section{Acknowledgements}

The author would like to thank Jeremy Butterfield, Adam Caulton, John Earman, Hans Halvorson and Miklos Redei for helpful discussions and comments.

\section{References}

Bell, J. S. (1975). The theory of local beables. In Speakable and unspeakable in quantum mechanics (pp. 52-62). Cambridge: Cambridge University Press.

Berkovitz, J. (1998a). Aspects of quantum non-locality. Studies in History and Philosophy of Modern Physics, 29B, 183-222.

Berkovitz, J. (1998b). Aspects of quantum non-locality II. Studies in History and Philosophy of Modern Physics, 29B, 509-546.

Butterfield, J. (2007). Reconsidering relativistic causality. International Studies in the Philosophy of Science, 21, 295-328.

Clifton, R., \& Halvorson, H. (2000). Generic Bell correlation between arbitrary local algebras in quantum field theory. Journal of Mathematical Physics, 41, 1711-1717.

Clifton, R., \& Halvorson, H. (2001). Entanglement and open systems in algebraic quantum field theory. Studies in History and Philosophy of Modern Physics, 32, 131 .

Dimock, J. (1982). Dirac quantum fields on a manifold. Transactions of the American Mathematical Society, 269, 133-147.

Doplicher, S., Haag, R., \& Roberts, J. E. (1971). Local observables and particle statistics I. Communications in Mathematical Physics, 23, 199-230.

Doplicher, S., Haag, R., \& Roberts, J. E. (1974). Local observables and particle statistics II. Communications in Mathematical Physics, 35, 49-85.

Earman, J. (2014). No superluminal propagation for classical relativistic and relativistic quantum fields. Studies in History and Philosophy of Modern Physics,

under review.

Earman, J., \& Valente, G. (2014). Relativistic causality in algebraic quantum field theory. International Studies in the Philosophy of Science, 28, 1-48.

Epstein, H., Glaser, V., \& Jaffe, A. (1965). Nonpositivity of the Energy Density in Quantized Field Theories. Nuovo Cimento, 36, 1016-1022.
Fleming G. (2000). Reeh-Schlieder meets Newton-Wigner, In Philosophy of science, (Vol. 67 (3, suppl.), pp.495-515) (PSA 1998, Part II (Kansas City, MO))

Fleming, G., \& Butterfield, J. (1999). Strange positions. In: J. Butterfield, \& C. Pagonis (Eds.), From physics to philosophy (pp. 108-165). New York: Cambridge University Press.

Fredenhagen, K. (1985). A remarks on the cluster theorem. Communications in Mathematical Physics, 97, 461-463.

Geroch, B. (2011). Faster than light? 〈http://arxiv.org/abs/1005.161〉.

Haag, R. (1992). Local quantum physics (2nd ed.). New York: Springer.

Haag, R., \& Schroer, B. (1962). Postulates of quantum field theory. Journal of Mathematical Physics, 3, 248-256.

Hellwig, K. E., \& Kraus, K. (1969). Communications in Mathematical Physics, 11, 214.

Hellwig, K. E., \& Kraus, K. (1970). Communications in Mathematical Physics, 16, 142

Horuzhy, S. S. (1990). Introduction to algebraic quantum field theory. Dordrecht: Kluwer.

Jones, M., \& Clifton, R. (1993). Against experimental metaphysics. In: P. French (Ed.), Midwest studies in philosophy, Vol. 18 (pp. 295-316). South Bend, Indiana: University of Notre Dame Press.

Kadison, R. V., \& Ringrose, J. R. (1997). Fundamentals of the theory of operator algebras, (Vol. I). Providence, RI: American Mathematical Society.

Kraus, K. (1983). States, effects and operations. In Lecture notes in physics (Vol. 190). New York: Springer.

Maudlin, T. (1994). Quantum non-locality and relativity: Metaphysical intimations of modern physics. Oxford: Basil Blackwell.

Rédei, M., \& Summers, S. J. (2008). When are quantum systems operationally independent? International Journal of Theoretical Physics, 49, 3250-3261.

Rédei, M., \& Valente, G. (2010). How local are local operations in local quantum field theory? Studies in the History and Philosophy of Modern Physics, 41, 346-353.

Redhead, M. (1995). More ado about nothing. Foundations of Physics, 25, 123-137.

Reeh, H., \& Schlieder, S. (1961). Bemerkungen zur unitaraquivalenz von Lorentzinvarianten Feldern. Nuovo Cimento, 22, 1051-1068.

Schlieder, S. (1968). Einige Bemerkungen zur Zust ändsanderung von relativistischen quantenmeehanischen Systemen durch Messungen und zur Lokalitäts-

forderung. Communications in Mathematical Physics, 7, 305-331.

Segal, I. E., \& Goodman, R. W. (1965). Anti-locality of certain Lorentz-invariant operators. Journal of Mathematics and Mechanics, 14, 629-638.

Summers, S. (1990). On the independence of local algebras in quantum field theory. Reviews in Mathematical Physics, 2, 201-247.

Summers, S. (2009). Subsystems and independence in relativistic microscopic physics. Studies in the History and Philosophy of Modern Physics, 40, 133-141.

Summers, S., \& Werner, R. (1987). Bell's inequalities and quantum field theory, I: General setting. Journal of Mathematical Physics, 28, 2240-2247.

Summers, S., \& Werner, R. (1988). Maximal violation of Bell's inequalities for algebras of observables in tangent spacetime regions. Annales de l'Institut Henri Poincaré. Physique Théorique, 49, 321-334.

Valente, G. (2013). Local disentanglement in relativistic quantum field theory. Studies in History and Philosophy of Modern Physics, 44, 424-432.

Weinstein, S. (2006). Superluminal signaling and relativity. Synthese, 148, 381-399. 